\author{
I J M I \\ International Journal of Machine Intelligence \\ ISSN: 0975-2927 \& E-ISSN: 0975-9166, Volume 3, Issue 3, 2011, pp-150-154 \\ Available online at http://www.bioinfo.in/contents.php?id=31
}

\title{
BIOMETRIC IDENTIFICATION OF INDIVIDUAL USING STATISTICAL PROPERTIES OF PALM
}

\section{RAUT SHRIRAM DAGADU AND MANZA R.R.*}

Department of CS \& IT , Dr.B.A.M.University Aurangabad, India

*Corresponding author. E-mail: Shriram.raut@yahoo.in, manzaramesh@gmail.com

Received: September 29, 2011; Accepted: November 03, 2011

\begin{abstract}
This paper proposes a Biometric Palm Identification of Individual using Statistical Properties of palm. The proposed work discusses the significance; since both the palm print and hand shape images are proposed to extract from the single hand image acquired from a sensor. We can discuss the new approach for palm print recognition is based on Statistical properties such as Mean square Error, Signal to Noise Ratio, Standard deviation. The comparison and combination of proposed statistical properties can be evaluated by considering the various statistical aspects are Mean Square Error (MSE), Signal to Noise Ratio (SNR), Standard Deviation.
\end{abstract}

Keywords: Biometrics, PolyU_Palmprint_Database, enrollment, identification,; Palm print recognition

\section{Introduction}

Multimodal biometric systems have recently attracted the attention of researchers and some work has already reported in literature. Most of the reported work has bimodal biometric system such as Finger prints, Face recognition, Iris, Hand and Palm print recognition. The biometric use of palm prints uses ridge patterns to identify an individual [1]. Palms of hands epidermal ridges, thought to provide a friction surface to assist with gripping an object on surface [2]. Palm print identification systems measure and compare ridges, lines and Minutiae found on the palm. Palm print recording and identification for law enforcement purposes has been in existence almost as long as palm prints systems are reported to comprise $30 \%$ of all crime scene marks.

As much as another $20 \%$ are made up of the edge of the hand, fingers between the palm and fingertips and other parts of the hand. A key driver for law enforcement agencies to adopt full-hand scan technologies is the high incidence of hand related crime scene marks [2]. Joao de Barros, an early explorer and writer, wrote that the Chinese merchants distinguished young children from each other by recording palm prints on paper with ink. One of the earliest AFIS systems built to support palm prints is believed to have been developed in Hungary in the early 1990's. In 1997, the technology was bought by a US company. In recent years, most AFIS vendors have added palm print records capabilities to their systems.[4]

\section{Methods used in Recognition}

There are three groups of marks which are used in palm print identification: Geometric features, such as the width, length and area of the palm. Geometric features are a coarse measurement and are relatively easily duplicated. In themselves they are not sufficiently distinct[3].

Line features principal lines and wrinkles. Line features identify the length, position, depth and size of the various lines and wrinkles on a palm. While wrinkles are highly distinctive and are not easily duplicated, principal lines may not be sufficiently distinctive to be a reliable identifier in themselves; and Point features or minutiae. Point features or minutiae are similar to fingerprint Minutiae and identify, amongst other features, ridges, ridge endings, bifurcation and dots[3].

Palm creases and ridges are often superimposed which makes feature extraction difficult. An important issue in palm print recognition is to extract palm print features that can discriminate an individual from the other. There are two popular approaches to palm print recognition. One of the approaches is to transform palm print images into specific transformation domains [2].

Palm print authentication is one of the relatively new physiological biometric technologies which exploit the unique features on the human palm print, namely principle lines, wrinkles, ridges, datum points, etc [3].

\section{Statistical Properties}

Statistics is the science of the collection, organization, and interpretation of data. It deals with all aspects of this, including the planning of data collection in terms of the design of surveys and experiments.[8]

The basic step of a statistical Experiment is:

1. Planning the research, including finding the number of replicates of the study, using the following information: Preliminary estimates regarding the size of treatment effects, alternative hypotheses, and the estimated 
experimental variability. Consideration of the selection of experimental subjects and the ethics of research is necessary. Statisticians recommend that experiments compare (at least) one new treatment with a standard treatment or control, to allow an unbiased estimate of the difference in treatment effects.

2. Design of experiments, using blocking to reduce the influence of confounding variables, and randomized assignment of treatments to subjects to allow unbiased estimates of treatment effects and experimental error. At this stage, the experimenters and statisticians write the experimental protocol that shall guide the performance of the experiment and that specifies the primary analysis of the experimental data.

3. Performing the experiment following the experimental protocol and analyzing the data following the experimental protocol.

4. Further examining the data set in secondary analyses, to suggest new hypotheses for future study.

5. Documenting and presenting the results of the study. Statistics rarely give a simple Yes/No type answer to the question asked of them.

Interpretation often comes down to the level of statistical significance applied to the numbers and often refers to the probability of a value accurately rejecting the null hypothesis (sometimes referred to as the p-value) [8].

Referring to statistical significance does not necessarily mean that the overall result is significant in real world terms. For example, in a large study of a drug it may be shown that the drug has a statistically significant but very small beneficial effect, such that the drug will be unlikely to help the patient in a noticeable way.

\section{Feature Selection}

In our work the feature selection is based on the statistical measurements of a palm for Palm print Recognition System. There are various statistical measurements out of which the measurements which were the part of our work and mainly want to focus IsMean Square Error (MSE), Signal to Noise Ratio, Standard Deviation.

There are various statistical measurement out of which the measurements which were the part of our work and mainly want to focus were-

- Mean Square Error (MSE).

- $\quad$ Signal to Noise Ratio (SNR).

- Standard Deviation.

And can be described as follows:

\section{Mean Square Error (MSE)}

The mean square error or MSE of an estimator is one of many ways to quantify the difference between an estimator and the true value of the quantity being estimated. [9]

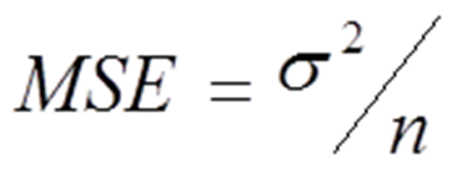

(1)

Mean squared error is sometimes used to refer to residual sum of squares, divided by the number of degrees of freedom. For an unbiased estimator, the MSE is the variance. Like the variance, MSE has the same unit of measurement as the square of the quantity being estimated.

The MSE of an estimator $\hat{\theta}$ with respect to the estimated parameter $\theta$ is defined as

$$
\operatorname{MSE}(\hat{\theta})=E\left[(\hat{\theta}-\theta)^{2}\right]
$$

The difference occurs because of randomness or because the estimator doesn't account for information that could produce a more accurate estimate.

The MSE is the second moment (about the origin) of the error, and thus incorporates both the variance of the estimator and its bias.

For an unbiased estimator, the MSE is the variance. Like the variance, MSE has the same unit of measurement as the square of the quantity being estimated. The standard error of the mean (SEM) is the standard deviation of the sample mean estimate of a population mean. (It can also be viewed as the standard deviation of the error in the sample mean relative to the true mean, since the sample mean is an unbiased estimator). SEM is usually estimated by the sample estimate of the population standard deviation (sample standard deviation) divided by the square root of the sample size (assuming statistical independence of the values in the sample):

$$
S D_{X}=\frac{\sigma}{\sqrt{n}}
$$

Where, ' $\sigma$ ' is the sample standard deviation (i.e., the sample based estimate of the standard deviation of the population), and ' $n$ ' is the size (number of observations) of the sample.

\section{Signal to Noise Ratio}

The Signal to Noise Ratio (SNR) is as the reciprocal of the coefficient of variation, i.e. the ratio of mean to standard deviation of a signal or measurement-

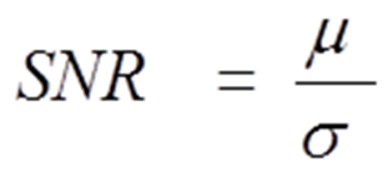

(4)

Where ' $\mu$ ' is the signal mean or expected value and ' $\sigma$ ' is the standard deviation of the noise, or an estimate thereof [10]. Thus it is commonly used in image processing, where the SNR of an image is usually 
calculated as the ratio of the mean pixel value to the standard deviation of the pixel values over a given neighborhood.

\section{Standard Deviation}

Standard deviation is a widely used measurement of variability or diversity used in statistics and probability theory. [8] It shows how much variation or "dispersion" there is from the "average" (mean or expected/budgeted value).A low standard deviation indicates that the data points tend to be very close to the mean, whereas high standard deviation indicates that the data is spread out over a large range of values.

Technically, the standard deviation of a statistical population, data set, or probability distribution is the square root of its variance.

An estimator for $\sigma$ sometimes used is the standard deviation of the sample, denoted by $S$ and defined as follows:

$$
S=\sqrt{\frac{1}{N} \sum_{i=1}^{N}\left(x_{i}-\bar{x}\right)^{2}}
$$

This estimator has a uniformly smaller mean squared error than the sample standard deviation and is the maximum-likelihood estimate when the population is normally distributed [10].

But this estimator, when applied to a small or moderately sized sample, tends to be too low: it is a biased estimator.

\section{Work - Flow \\ Enrollment Phase}

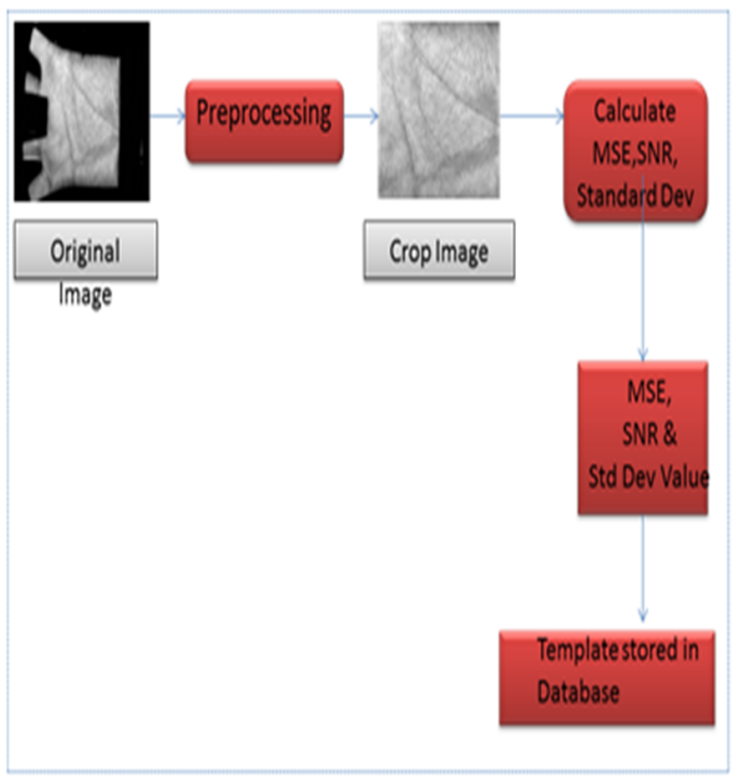

Fig. 1- Enrollment Methodology

\section{Verification Phase}

Bioinfo Publications

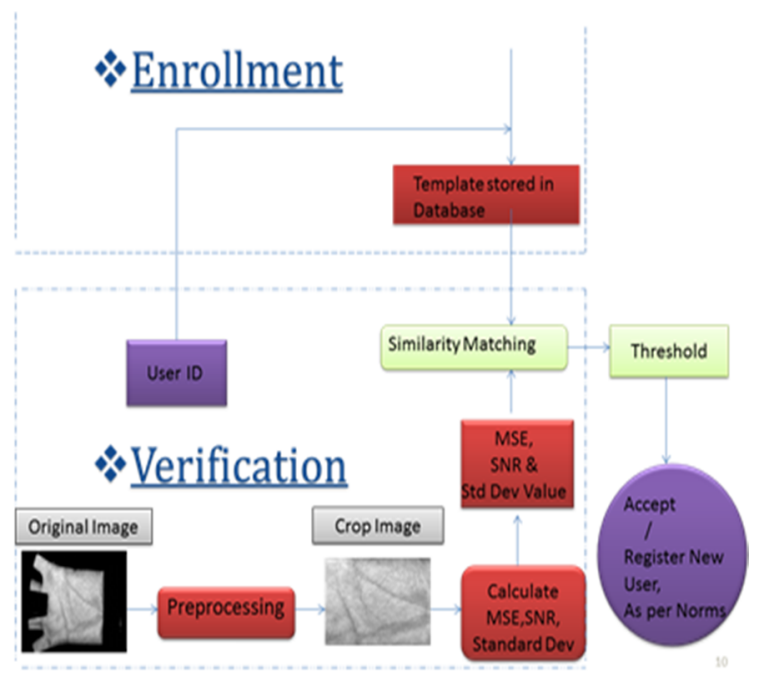

\section{Verification Methodology}

\section{About Database}

The Hong Kong Polytechnic University (PolyU) 2D_Palmprint Database [11]. Palm print has proved to be one of the most unique and stable biometric characteristics. Almost all the current palm print recognition techniques capture the two dimensional (2D) image of the palm surface and use it for feature extraction and matching. Although 2D palm print recognition can achieve high accuracy [11]. The PolyU 2D Palm print Database contains 7680 samples collected from 384 different palms. Twenty samples from each of these palms were collected in two separated sessions, where 10 samples were captured in each session, respectively. The average time interval between the two sessions is one month. The all palm print images are of same size and same dimension such as $384 \mathrm{X}$ 284. [11] And each 2D palm print image was recorded in BMP format image file.

The palm print images have a name sequence and can be interpreted as follows-

e.g. - A palm print image name "PolyU_001_F.bmp" can be interpreted as the initiated word 'PolyU' is the copyright for the Polytechnic University of Hong Kong. Then followed '001' indicate the subject enrollment number as it varies with person to person palm print image.

The followed ' $F$ ' indicate the session enrollment that whether it is the First or Second. And finally the format 'BMP' of the image file.

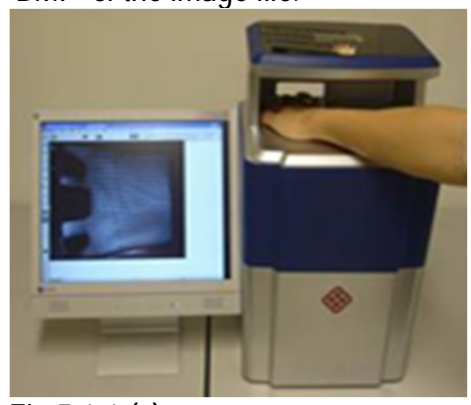

Fig.5.1.1 (a) 

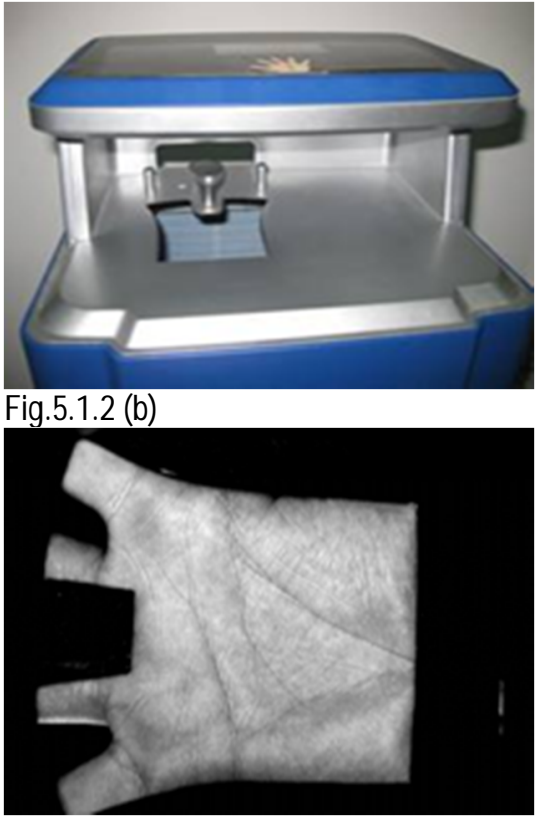

Fig.5.1.3 (c)

Fig.5.1.1 (a) the outlook of image acquisition device; Fig.5.1.2 (b) the device is being used to collect palm print image;

Fig.5.1.3(c) The "PolyU_001_F_01.bmp" palm print image. [11]

\section{Experiment and Result}

The Experiment was performed over the palm Image taken from the database developed by the Hong Kong Polytechnic University (PolyU) palm print database.

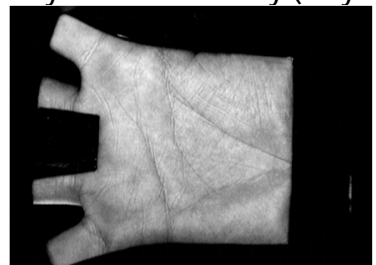

Fig.6.1.1(a) PolyU_001_F_01.bmp palm image from PolyU_Palmprint_Database(2nd version). [8]

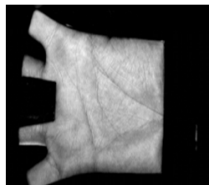

fig.6.1.2(b)

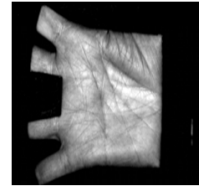

fig.6.1.3(c)

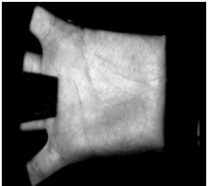

fig.6.1.4(d)

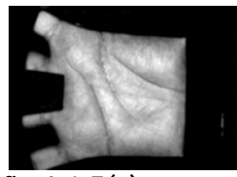

fig.6.1.5(e)

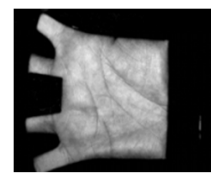

fig.6.1.1(f)
Compared Image [8] of various palm image Are as follows-

fig.6.1.2 (b) PolyU_Database_001_F,
Fig.6.1.3(c) PolyU_Database_003_F, fig.6.1.4 (d) PolyU_Database_005_F, fig.6.1.5 (e) PolyU_Database_007_F, fig.6.1.6 (f) PolyU_Database_009_F.

These calculated values are distinct for each and every individual. So a palm matching can be done on the basis of individual statistical palm properties. And can be used to identify an individual. The testing image who's MSE, SNR and standard deviation values is being calculated as we input the testing image. The corresponding one to one matching has to be done by comparing it with our already trained samples. The corresponding matching sample of testing palm sample will be got matched depending upon the similar MSE, SNR and standard deviation values of the Palm image. Hence the Biometric identification of individual can be done on the basis of its palm statistical properties such MSE, SNR and standard deviation.

\section{Result}

The MSE, SNR and standard deviation values of the right palm image is being calculated. The statistical (MSE, SNR and std dev) values of palm are individually distinct from person to person. And can used to as comparison aspect to identify an individual. We can use the multimodal statistical values for matching various palm image templates. The Perfect Match can be drawn by matching a testing palm image sample with the trained sample depending upon the MSE, SNR and standard deviation values. The FRR, FAR and TSR are as follows.

\section{Conclusion}

The biometric use of palm prints uses ridge patterns to identify an individual. Palms of hands epidermal ridges, thought to provide a friction surface to assist with gripping an object on surface. Palm print identification systems measure and compare ridges, lines and Minutiae found on the palm. In our work the feature selection is based on the statistical measurements and properties of a palm for Palm print Recognition System. There are various statistical measurements out of which the measurements which were the part of our work and mainly want to focus Is-Mean Square Error (MSE), Signal to Noise Ratio (SNR) and Standard deviation. The experiment is carried over the PolyU_2D palm print database. The experimental values were drawn from the experiment that was based on various MSE values, SNR values and Standard Deviation values of palm prints taken from the PolyU_2D palm print Database. The MSE acts as the linear classifiers and the identification system can be made multimodal by applying the other supportive properties such as SNR and Standard Deviation. Thus we can state that the statistical property can be considered as the features to identify an individual.

\section{Acknowledgement}

We are very much thankful for Dept. of CS \& IT, Dr. B.A.M.U. Aurangabad, and India for providing a great support to make such kind of research over Biometric Identification by using palm and its statistical properties. We are also thankful to the 
staff and research student of this department to highlight many things during our research.

\section{References}

[1] Chin-Chuan Han, Hsu-Liang Cheng, KuoChin(2002) Personal Authentication Using Palm Print Features

[2] Xiangqian Wu, Kuanquan Wang (2004) A Novel Approach of Palm-line Extraction

[3] Ajay Kumar, David Zhang(2005) Personal Authentication using multiple palm print Representation.

[4] Koichi Ito,Takafumi Aoki, Hiroshi Nakajima,Koji Kobayashi and Tatsuo Higuchi (2006) A palmprint recognition algorithmusing phasebasedimage matching

[5] Anil K. Jain, Jianjiang Feng(2009)Latent Palm matching.

[6] Ajay Kumar, David Zhang (2006)Personal Recognition Using Hand Shape and Texture.

[7] Anil K. Jain, Jianjiang Feng(2009) Pattern Analysis And Machine Intelligence.

[8] Jamie DeCoster ( 1998) Introductory Statistics Notes.
[9] Lie-Liang Yang(2009) Minimum Mean-Square Error And Maximum Likelihood Multiuser Detection:Statistical Properties and Applications

[10] Keone Hon, Text-book: An Introduction to Statistics.

[11] Harvey Motulsky,1999-2005, TextBook:Statistics Guide, Statistical analyses for laboratory and clinical Researcher.

[12] Chris Roberts(2006) Biometric Technologies Palm and Hand.

[13] Xingjian Wu, David Zhang, Kuanquan Wang(2003)Fisher palms based palm print recognition

[14] Theodoridis S., Koutroumbas K., TextBook:Pattern Recognition

[15] Gregory Mendell, Michael Landry(2005) Stack Slide and Hough Search SNR and Statistics LIGO.

[16] Li W., Zhang D., Zhang L., Lu G. and Yan J. The Hong Kong Polytechnic University (PolyU) Palm Print Database

[17] Richard O. Duda, Peter E. Hart, David G. Stork(2000) Pattern Classification.

[18] James F., Cousins R., Cowan G.(2005) TextBook- STATISTICS.

Table 6.1.1: - The calculated MSE, SNR and Standard Deviation.

\begin{tabular}{|r|l|r|r|r|}
\hline Sr. No. & Right palm image & \multicolumn{1}{l|}{$\begin{array}{l}\text { MSE } \\
\text { value }\end{array}$} & \multicolumn{1}{l|}{ SNR value } & Std Dev value \\
\hline 1 & PolyU_001_F_01.bmp & 0.0175 & 8.4678 & 21.5897 \\
\hline 2 & PolyU_003_F_01.bmp & 0.0328 & 6.0436 & 29.517 \\
\hline 3 & PolyU_005_F_01.bmp & 0.0865 & 3.7427 & 47.9331 \\
\hline 4 & PolyU_007_F_01.bmp & 0.0321 & 6.4637 & 29.1923 \\
\hline 5 & PolyU_009_F_01.bmp & 0.0251 & 6.8626 & 25.8358 \\
\hline 6 & PolyU_011_F_01.bmp & 0.0136 & 9.7804 & 18.979 \\
\hline 7 & PolyU_013_F_01.bmp & 0.0211 & 8.0022 & 23.6798 \\
\hline 8 & PolyU_015_F_01.bmp & 0.0283 & 6.73 & 27.4457 \\
\hline 9 & PolyU_017_F_01.bmp & 0.0208 & 8.0811 & 23.4864 \\
\hline 10 & PolyU_019_F_01.bmp & 0.032 & 6.8891 & 29.1825 \\
\hline 11 & PolyU_021_F_01.bmp & 0.0279 & 6.8241 & 27.2462 \\
\hline 12 & PolyU_023_F_01.bmp & 0.0222 & 7.8497 & 24.3128 \\
\hline 13 & PolyU_025_F_01.bmp & 0.0237 & 7.5438 & 25.0819 \\
\hline 14 & PolyU_027_F_01.bmp & 0.0223 & 7.6621 & 24.3341 \\
\hline 15 & PolyU_029_F_01.bmp & 0.0338 & 6.86 & 29.9894 \\
\hline
\end{tabular}

Table 7.1.1:- The FRR, FAR and TSR rates Over an experiment.

\begin{tabular}{|l|c|c|c|}
\hline & Mean Square error & $\begin{array}{c}\text { Signal to Noise } \\
\text { Ratio }\end{array}$ & $\begin{array}{c}\text { Standard } \\
\text { Deviation }\end{array}$ \\
\hline FRR & $0.00 \%$ & $0.00 \%$ & $0.00 \%$ \\
\hline FAR & $0.00 \%$ & $0.00 \%$ & $0.00 \%$ \\
\hline TSR & $100 \%$ & $100 \%$ & $100 \%$ \\
\hline
\end{tabular}

